tigere Uebersetzung „Buch der Glaubenssätze und philosophischen Lehren ${ }^{\star 25}$ ) verdrängt sein sollte.

Budapest, 31. December 1882.

\title{
Ueber den Einflufs des Accentes auf die Vocalentfaltung nach Gutturalen.
}

\section{Von Franz Prätorius.}

Dals der Accent in einer gewissen Beziehung steht zur Entwicklung secundärer Vocale nach Gutturalen, ist erkannt von Ewald, Lehrb. d. hebr. Spr. ${ }^{8} \S 60$ a : Weil sich Silben vor dem Tone ,im übergange zum folgenden leicht öffnen und lösen können", kann in solchen Silben vor dem Tone „ein flüchtiger vocal nach dem hauchlaute sich eindrängen." Vgl. § $28 \mathrm{~b} \mathrm{~b}), \S 65 \mathrm{~d}$. Dals dagegen in und nach dem Tone ein solches Nachballen und Hinüberschleifen nicht möglich ist, steht klar ausgesprochen $\S 61$ (namentlich der kürzeren Ausgabe von 1874). Demgegenüber kann ich nicht finden, dafs Ewald sich deutlich da-

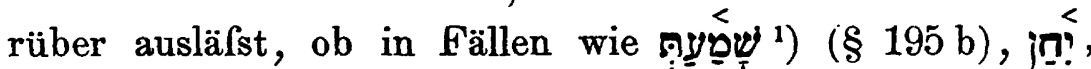

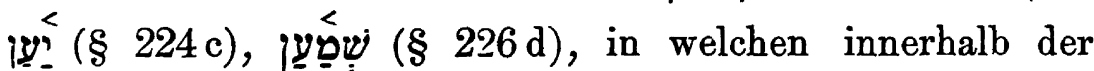
Tonsilbe nach dem Guttural ein secundärer Vocal entstanden ist, Ausnahmen von seiner Regel vorliegen, oder ob der secundäre Vocal sonst wie zu erklären ist.

${ }^{25}$ ) S. K a u fmann, Attributenlehre, S. 250 f.

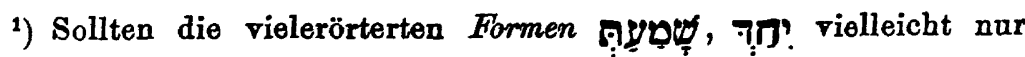
auf Zwitterschreibungen beruhen der Formen

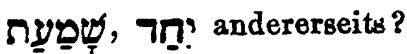


Auch bei Gesenius ${ }^{23} \S 22,4$ und $\S 65,2$ ist der Einflufs des Accentes auf die Vocalentfaltung nach Gutturalen in gleichem Sinne kurz angedeutet, eine Erklärung desselben indefs nicht versucht worden. In den oben aufgeführten drei besonderen Fällen ist der secundäre Vocal dann folgerecht erklärt äls Hülfsvocal zur Vermeidung des doppeltconsonantischen Wortschlusses, nicht etwa als hervorgerufen durch den Gutturalen $(\S 65,2, \S 75$ Anm. $3 \mathrm{~d}$, $\S 46$ Anm. 3). Ebenso scheint sich Philippi die Sache zu denken, ZDMG XXXII S. 92 Zl. 17. Bei dieser Auffassung bleibt aber das grofse Bedenken, dafs die nicht gutturalischen Parallelen des ersten Falls stets, die des zweiten oft des Hülfsvocals entbehren, während die gutturalhaltigen Beispiele des ersten Falls nur selten, die des zweiten nie 1) ohne secundären Vocal sind. Die Ungleichheit wird also doch auf Rechnung des Gutturals zu schreiben sein. Ueber den dritten Fall läfst sich aus Mangel in Betracht kommender Beispiele nichts sagen.

Bei Böttcher $\S 400,401,1050$, Olshausen $\S 232$ l, König, Lehrgeb. I S. 295 finden sich nur flüchtige Andeutungen über eine Beziehung des Accents zur. Vocalentfaltung nach Gutturalen. Die übrigen mir vorliegenden Grammatiken schweigen, soviel ich sehe, vollständig von einer derartigen Beziehung. -

Es ist meines Erachtens nicht der Accent an sich, welcher die Entstehung eines secundären Vocals nach einer von ihm getroffenen gutturalisch ausgehenden Silbe unter allen Umständen verhindert, sondern es ist die Stellung des Accents, welche die Entstehung einer neuen Silbe eventuell verbietet. $\mathrm{Da}$ nach der jetzigen Gewohnheit zu betonen im Hebr. der Accent an die Ultima oder die Penultima gebunden ist, so mufs die Sprache naturgemäls vermeiden, eine betonte Penultima durch secundäre Silben-

1) Vgl. indefs die vorige Anmerkung. 
wucherung zur Antepenultima zu machen. Dies scheint

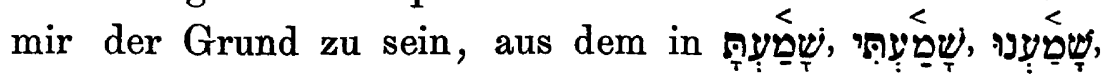

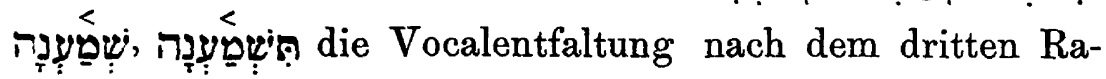
dical nicht stattgefunden hat, weil nämlich in u. s. w. der Accent die Antepenultima treffen würde. In

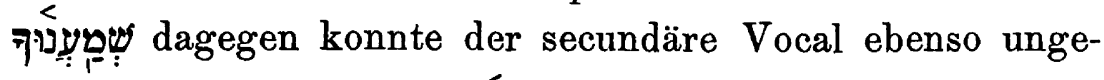

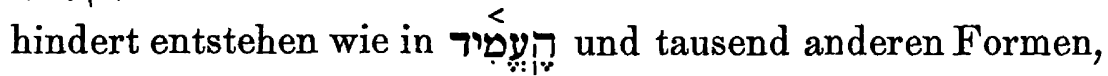
in denen die Stellung des Accents zum Wortende nicht gestört wurde. Wenn sich die 2. Pers. Plur. Perf. von den Verbis ult. gutt. nur mit dem ursprünglichen Silbenbau findet, obwohl wir nach dem eben Ausgeführten auch שְִׁ erwarten könnten, so mag daran der entsprechende Singular שָ schuld sein (vgl. auch Philippi in Steinthal's Zeitschrift X 279 Anm. 1). Auch bei der alten Contextbetonung, welche sich nach 1 conversivum eventuell erhalten hat, könnten wir mit secundärem Vocal erwarten lassen, ob solche Formen nicht wirklich existirt haben und erst später durch den Einflufs der sonst herrschend gewordenen Formen mit pausaler Betonung umgestaltet worden sind. Uebrigens hat ja auch sonst die Sprache den secundären Vocal nicht immer entwickelt, wenngleich von Seiten des Accentes kein Hindernil's entgegensteht.

Dem Widerwillen gegen die Betonung der Antepenul-

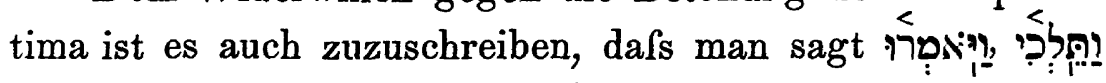

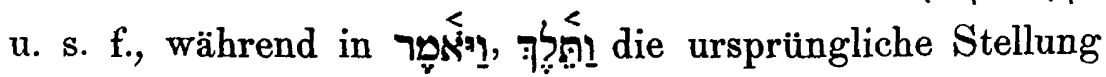
des Accentes aus alter Zeit her beibehalten werden durfte, da die Antepenultima hier durch Schwinden des auslautenden Vocals zur Penultima geworden war (grade so wie in

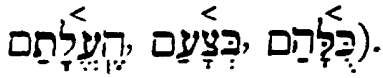

Nun wird es klar sein, warum in den eingangs angeführten besonderen drei Fällen der secundäre Vocal nach 
dem Guttural entstehen konnte, obgleich die betreffende Silbe den Accent hat : Der Accent wird in seiner Stellung zum Wortende nicht in ungewohnter Weise gestört; er traf bisher die Ultima, nach der neuen Silbenbildung trifft er die Penultima, was den Accentgesetzen durchaus noch conform ist. -

Man wird einwenden, dals die Betonung der Antepenultima auch der jetzigen Gewohnheit des Hebr. durchaus nicht fremd sei, sobald nur die Penultima den kürzesten Vocal, Schwa mobile habe. Diese Art der Betonung finde sich am häufigsten beim נסוג אחור, dann bei einer Reihe mit Suffixen bekleideter Formen, endlich auch bei Antritt des $\pi$ locale. - Demgegenüber würde zu bemerken sein, dals das Hebr. allerdings zwei in engem Context unmittelbar zusammentreffende Hauptaccente nicht liebt, und dafs zur Vermeidung dieses Zusammenstofses der Accent des ersteren Wortes eventuell auf einen Gegenton 1) der Antepenultima oder gar der viertletzten Silbe verlegt wird. Wenn also in der That Betonungen vorkommen wie

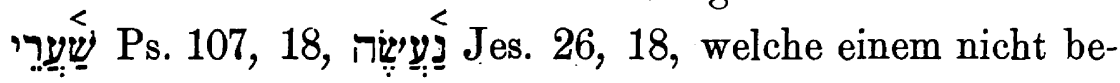

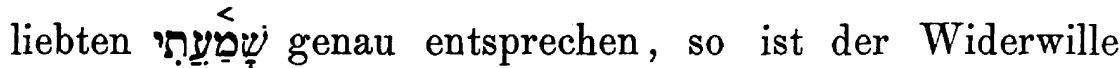
gegen jene Betonungen eben nur in bestimmtem Falle 'durch einen anderen, noch gröfseren Widerwillen überwunden, und jene Betonungen sind nicht schlechthin möglich.

Weiter zeigen allerdings einige mit Suffixen beklei-

1) Dafs die dritt- oder viertletzte Silbe ohnehin einen Gegenton haben mufs, um bei rückweichendem Accent den Hauptaccent aufnehmen zu können, ist, soviel ich sehe, nothwendig. Daher sind sowohl Betonungen wie wie רִֹ weil weder auf das kurze a, noch auf die Copula ; ein Gegenton fällt (vgl. Baer, Metheg-Setzung §§ 9. 12). - Dafs übrigens wirklich eine Zurückziehung, nicht ein Verharren.des ursprünglichen Hauptaccents vorliegt, ist wenigstens in Fällen, in denen das erstere Wort ein Status constructus ist, sicher. 
dete Formen z. T. in gehobener Rede noch den alten Accent. Es sind dies die beiden vereinzelten Formen

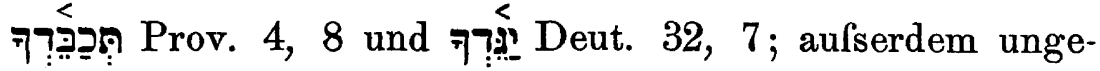

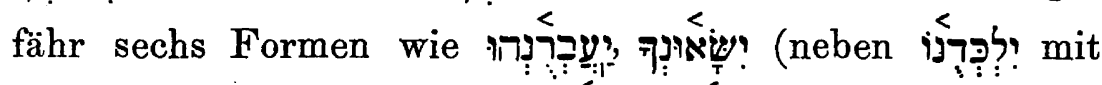

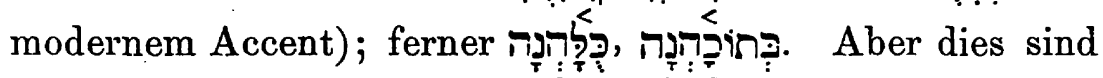
vereinzelte Reste, die von dem früheren Accentuationssystem übrig geblieben sind. - Nicht aber auf gleicher Linie mit den eben genannten Formen scheinen zu stehen

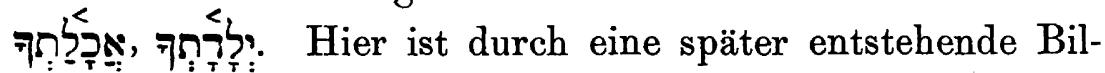
dung die Betonung der Antepenultima der Sprache aufgedrängt worden. Denn ursprünglich mufsten an die 3 . Pers. fem. sing. Perf. die Suffixe unmittelbar antreten, al-

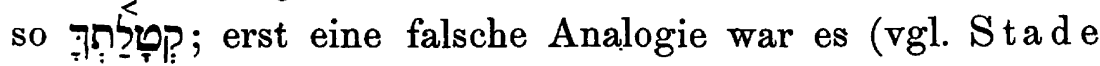
$\S 633 \mathrm{~b}$ ), welche später zwischen Verbalform und Suffix den Vocalanstofs einschob, zunächst unter Beibehaltung der älteren Accentsilbe, die nunmehr Antepenultima geworden. Indefs verursachte der Widerwillen gegen diese Betonung, dafs der Accent dieser neueren Bildung auch auf die Ul-

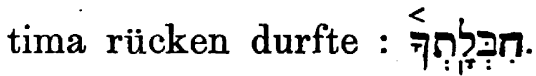

Das gleiche Schwanken, nur in noch stärkerem Mafse, tritt uns entgegen bei den Fällen mit $ה$ locale. Diese sind für unsere Frage ganz besonders instructiv. Der nominale Auslaut ă hat sich im Hebr. meines Erachtens, ebenso wie $\breve{\mathbf{l}}$ (oder $\hat{\imath}$ ?) und $\breve{\mathbf{u}}$ nur in derjenigen, im Nordsemitischen wenigstens seltneren Art des stat. constr. behaupten können, welche den vocalischen Auslaut überhaupt nicht völlig verschmähte (vgl. Schrader ABK 230, Philippi in theol. Literaturzt. 1881 Sp. 589), denn hier war er von jeher durch den Accent geschützt ${ }^{1}$ ). Es mufste somit aus

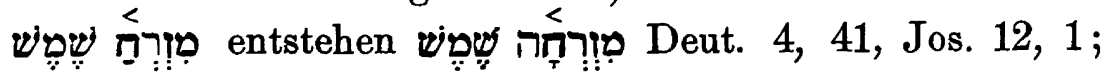

1) Dafs der hebr. Stat. constr. von jeher die Ultima betonte, wird mit der weit später auftretenden Contextbetonung der Ultima vermuthlich gleichen Grundes sein. 


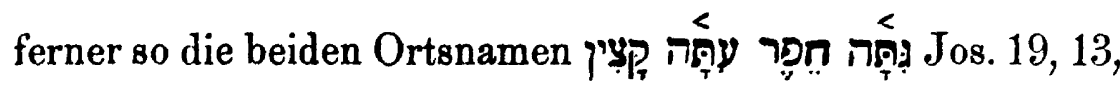
von denen der erstere 2 Kön. 14, 25 מ stat. absol. aber musfte das auslautende unbetonte ă aus rein lautlichem Grunde schwinden, und in der gewöhnlicheren Art des stat. const. fehlte es aus unbekannten Gründen schon seit ältester Zeit. Es erhielt sich für diese

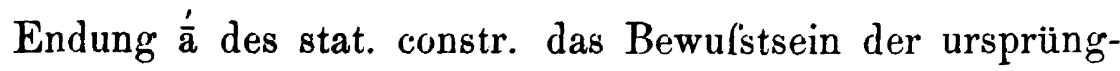
lichen Function als Zeichen des localen Casus, und dieses Bewulstsein rief das Bestreben wach, dem localen Casus auch dort, wo er abgestorben, also bei der gewöhnlichen Art des stat. constr. und beim stat. absol. von neuem Ausdruck zu verleihen. Und so ward denn dieses $\frac{\dot{a}}{\text { secundär }}$ auch auf den stat. absol. und auf die gewöhnlichere Art des stat. constr. übertragen. Diese Formen wurden dabei von der Sprache ganz unverändert auf der Stufe gelassen. zu der sie sich damals entwickelt hatten; es blieb ihnen auch ihr eigenthümlicher Accent, wodurch die ursprünglich

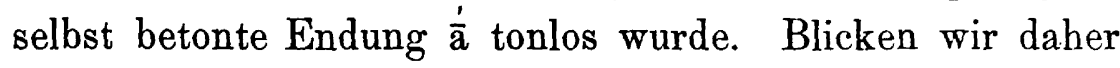
zunächst auf die gewöhnlichere Gattung des stat. constr.,

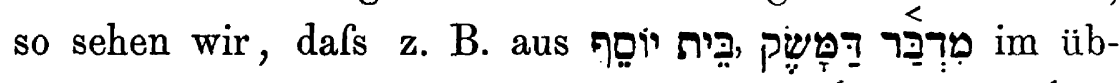

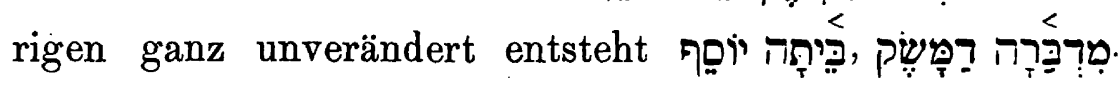

Die Secundärheit des Äntritts des localen à an den stat. absol. des Singulars springt deutlich in die Augen bei den Segolatformen. Es ist falsch, wenn bei Gesenius ${ }^{23} \S 90,2$, Anm. gesagt wird, dals die Segolatformen vor $\rightarrow$ locale in ihrer Grundform erscheinen, ohne Hülfsvocal. Nicht mehr die Grundform, nicht der einsilbige Stamm zeigt sich, sondern die bereits zweisilbig gemachte Secundärform. Natürlich mufs der kurze tonlose Vocal der zweiten Silbe bei der durch die Anfügung des $\bar{a}$ entstehenden Oeffnung derselben corrumpirt werden. Indem nun das locale $\bar{a}$ secundär an die fertig ausgebildete, auf der Penultima betonte Segolatform tritt, entsteht neuerdings ein Gebilde das den Accent auf der Antepenultima 
trägt, also auf derjenigen Silbe, welche $\mathrm{zu}$ betonen die Sprache nicht mehr gewohnt war. Hier zeigt sich nun deutlich der Kampf zwischen der neven Bildung, welche Betonung der Antepenultima verlangt, und dem neuen Accentuationsgesetz, welches Betonung der Antepenultima perhorrescirte. Die Segolatformen mit mittlerem $\mathrm{j}$ ertragen, soviel ich sehe, die Betonung der Antepenultima am leichtesten, gewils deshalb, weil in der Aussprache das $\breve{\text { e der }}$ Penultima von der diphtongisch klingenden Antepenultima

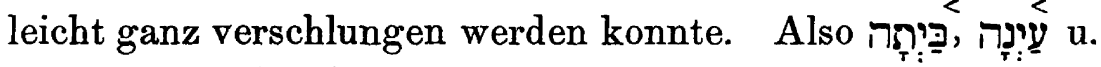
a. m., auf des $\bar{a}$ an das ursprüngliche Thema hätte

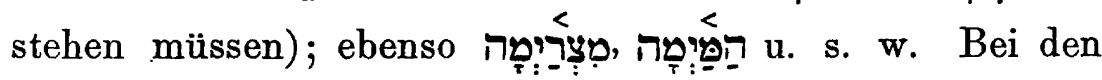
Segolatformen mit starkem mittleren Radical hat es indefs der Widerwille gegen die Betonung der Antepenultima zu Wege gebracht, dals zuweilen die Penultima ausgestofsen wurde, so dafs solche Formen, aufser Zusammenhang mit den übrigen Bildungen betrachtet, in der That den Eindruck machen können, als liege ihnen das ursprüngliche einsilbige Thema zu Grunde. Zuweilen wird der Betonung der Antepenultima auch in der Weise ausgewichen, dafs das locale $\bar{a}$ den Hauptaccent auf sich zieht und der Antepenultima nur den Gegenton lälst. Sehr häufig behält aber die Antepenultima den Hauptaccent. Auf diese Weise erklärt sich das Schwanken der Betonung und der Form bei den Segolatformen mit localem $\bar{a}$.

Beispiele der Betonung der Antepenultima sind : הָארקיָדה

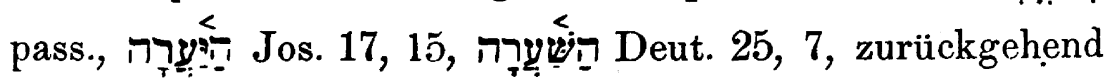

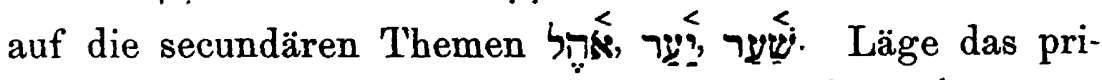

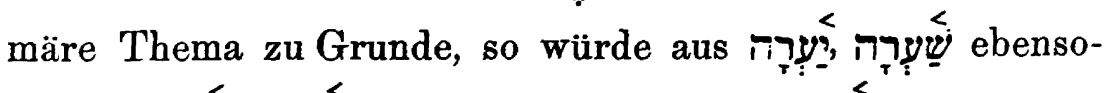

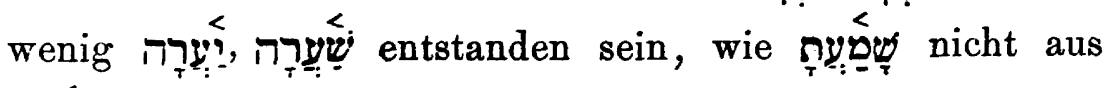

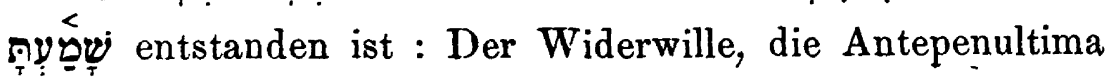


zu betonen hätte keine Vocalwucherung nach dem Guttural aufkommen lassen. Ist der mittlere Radical kein Guttural, so läfst es sich meist nicht erkennen, ob dreisilbig oder zweisilbig gesprochen werden soll; über m. ist in dieser Hinsicht nichts zu bestimmen; in Ps. 116, 14. 18 hat die gewöhnliche Ausgabe einmal 7, einmal 7, Baer-Delitzsch dagegen beidemal ausdrücklich 7 . - Reducirung zur Zweisilbigkeit liegt vor in dem

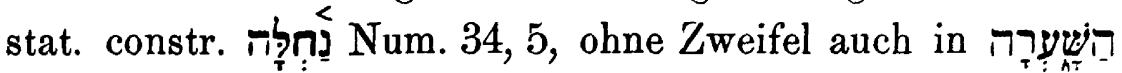
Deut. 22, 15, Jes. 28, 6; deutlich in pass. Hier kann

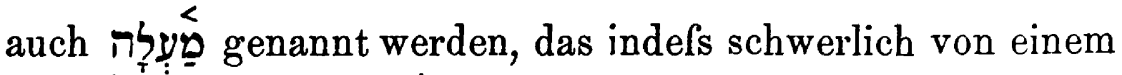

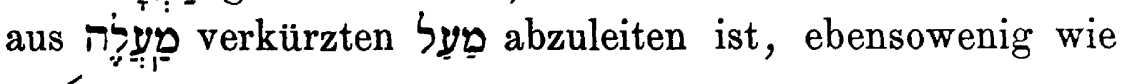

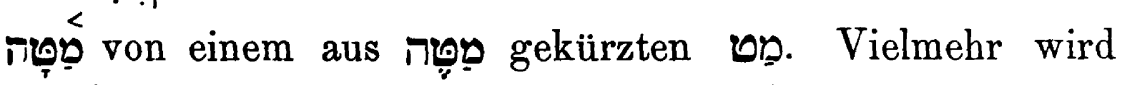

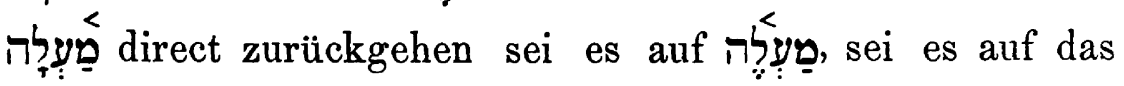

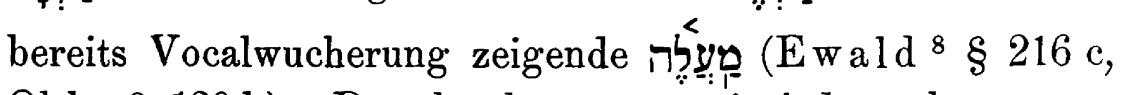
Olsh. $\S 130 \mathrm{~b}$ ). Das locale $\overline{\mathrm{a}}$ trat wie jede andere vocalische Endung nicht an das auslautende $\pi \%$, sondern an Stelle desselben, und zwar trat es tonlos an, wie immer in

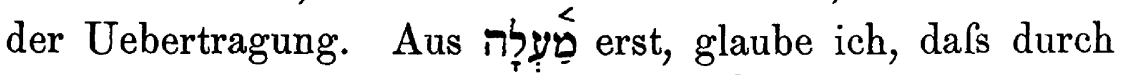
einen falschen Schlufs der Sprache صِ Die Antepenultima hat endlich den Accent an die Ultima

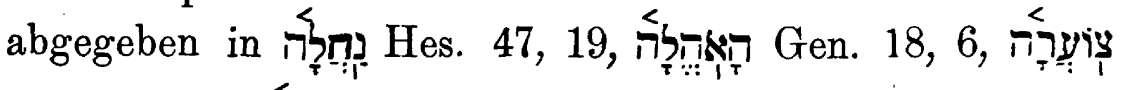
Gen. 19, 23, 兒 Mi. 4, 12. -

1) Vielleicht ist auf gleiche Weise auch wenn im Hebr. wirklich die vierradicalige Wurzel vorliegt, so sehe ich nicht, wie aus לִ לַילי לִ לילֶה hätte entstehen können, st. const. לִילֶ; der Plural konnte לִילוֹ bilden, wie er in der That heifst. Durch Antritt des localen à mufste entstehen לִ. Diese Form sah aber aus wie ein stat. constr. und wurde gewifs auch als

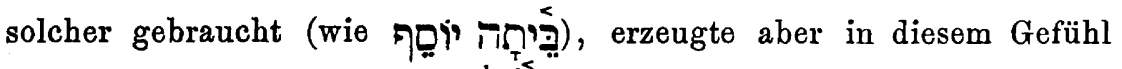

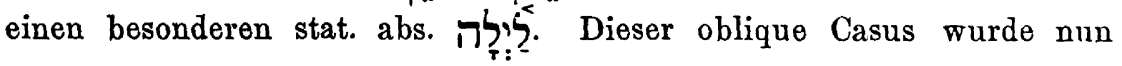
zwar auch an Stelle des Nomens schlechthin gebraucht, aber es entstand doch auch eine besondere Form לִיל st. constr. 
Mit diesem Einflufs des Accentes auf die Vocalentfaltung hängt es auch aufs engste zusammen, dafs es wohl Verba prim. gutt. $\aleph$ giebt, aber nicht ult. gutt. $\aleph$, wie dies gleichfalls Ewald § 75 ungefähr erkannt hat. Das relativ ursprüngliche Lautgesetz ist, dafs silbenschlielsendes $N$ seinen Consonantwerth verliert, ganz unbekümmert um die Accentverhältnisse. In späterer Zeit aber konnten sich solche Formen regeneriren unter der Einwirkung der Formen mit erhaltenem, silbenanlautendem $\mathbf{\aleph}$, und wesentlich unterstützt durch das inzwischen für die übrigen Gutturale ausgebildete Lautgesetz der Vocalentfaltung. Aber trotz

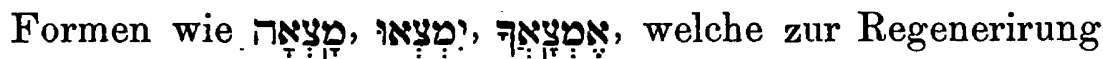
den Anstols hätten geben können, . trat dieselbe bei den Verbis ult. $\aleph$ doch nicht ein, weil dadurch wenn nicht in allen, so doch in dem gröfsten Theil der zu regenerirenden

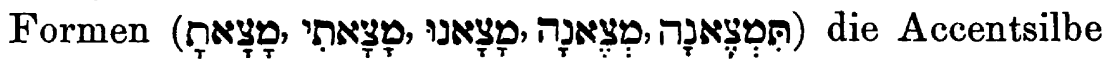
zur Antepenultima geworden wäre.

\section{Das Dodekapropheton der Alexandriner.}

Untersucht von Lic. Dr. K. Vollers.

\section{1) Einleitung ${ }^{1}$ ).}

Die Grundsätze, nach denen die Bearbeitung des vorliegenden kritischen Versuches erfolgt ist, sind im wesentlichen dieselben, zu denen der Verfasser sich in der Einleitung zu der Bearbeitung der sechs letzten Propheten der Sammlung (Berlin 1880) bekannte. Dort wurde als letztes

1) Nachstehende Einleitung ist als Halle'sche Inauguraldissertation bereits 1882 gedruckt.worden, jedoch nicht in den Buchhandel gekommen. Sie wird daher hier wiederholt. 